\title{
A Study of the Unified Scaling Law of Earthquakes in the Taiwan Region
}

\author{
Ching-Yi Tsai ${ }^{1}$ and Chiou-Fen Shieh ${ }^{1, *}$ \\ ${ }^{1}$ Institute of Seismology and Applied geophysics, National Chung-Cheng University, Chiayi, Taiwan, ROC
}

Received 2 March 2007, accepted 6 August 2007

\begin{abstract}
The unified scaling law (also called the BCDS model), merges: (1) Omori's Law, (2) Gutenberg-Richter's law, and (3) the geometrical fractal distribution of epicenters, all of which, in combination, investigate the occurrence of earthquakes from a spatial-temporal perspective. This study plans to verify important questions arising from the definition of the BCDS model by doing three experiments. Firstly, we examine the feasibility of applying this model to Taiwan using different cell sizes and cut-off magnitudes. Secondly, we ascertain the difference between aftershocks and main shocks in a unified scaling law by comparing earthquake time sequences with declustered ones. Thirdly, we investigate the differences among scaling relationships obtained from various geological settings in Taiwan.

Our results show that no matter how cell size and cut-off magnitude change, they produce a very similar pattern symbolizing the scaling law. Using a Z-map, after declustering, the constant part, which is an apparent indicator of the characteristics of aftershocks, disappears, and the slope of the fast decaying part, which corresponds to the main shock, remains almost the same. In addition, scaling laws obtained from four different sub-regions in Taiwan, although slightly different to each other, all show to be of a similar scaling law.
\end{abstract}

Key words: Scaling law, Fractal distribution, Earthquakes

Citation: Tsai, C. Y. and C. F. Shieh, 2008: A study of the unified scaling law of earthquakes in the Taiwan region. Terr. Atmos. Ocean. Sci., 19, 235-240, doi: 10.3319/TAO.2008.19.3.235(T)

\section{INTRODUCTION}

The nature of the spatial-temporal phenomenon of earthquake occurrences is a complicated one. It is easier as well as more important for seismologists to understand the correlation among earthquakes over a long period of time than to just study an individual isolated earthquake. Despite the complexity of the phenomenon, clues arising from studies must be examined in detail to better understand earthquake occurrence. Firstly, the relationship between time and earthquakes according to Omori's Law (Omori 1895) is such that the frequency of aftershocks decreases with time after a main shock, thereby precisely indicating the existence of a power law. Similarly, when we look at the relationship between earthquake occurrences from the point of view of time and space, the appearance of a power law can be deduced from the Gutenberg-Richter law (Gutenberg and Richter 1944). Accordingly, the frequency of earthquake

\footnotetext{
* Corresponding author

E-mail: seifent@eq.ccu.edu.tw
}

occurrence changes with the magnitude threshold of the earthquakes occurring in a certain area. These two powerlaw relationships between the waiting time and magnitude of earthquakes can be considered as a form of scale invariance in time and energy. This scale invariance is also shown in the spatial distribution of earthquakes. Hence, several seismologists (Okubo et al. 1987; Kagan and Jackson 1994; Marsan et al. 2000) consider spatial distributions of the epicenters of earthquakes to be fractal, though at times earthquakes may show significant deviation from this pattern.

Although earthquakes display complex spatial-temporal behavior, statistical evidence shows that several selfsimilarity properties can be found. Bak et al. (2002) and Christensen et al. (2002) pioneered an innovative model, called the BCDS model, to interpret the spatiotemporal distribution of earthquakes by merging: (1) Omori's Law, (2) the Gutenberg-Richter law, and (3) the geometrical fractal distribution of epicenters; thereby creating a unified scaling law. In brief, only a critical phenomenon exhibits a power 
law; that is, the hypothesis notes that earthquake occurrences can be considered self-organized critical (SOC) (Bak et al. 1988) phenomena.

The ingenious result of Bak et al. (2002) has raised other discussions and further research (Corral 2004a, b; Corral 2005; Lindman et al. 2005). Some scientists redefined the procedure of BCDS to investigate earthquakes in their own areas. In this way, we too introduce this theory to analyze and better understand earthquakes in Taiwan in the hope of getting closer to verifying the following issues: (1) how intrinsic characteristic seismicity in Taiwan is regulated by this scaling law; (2) how feasible is it to use this model in studying main and after shocks separately; and (3) this scaling law appropriate applied to Taiwan's complicated geological and tectonic structure.

\section{THEORY}

A two point spatial and temporal analysis was adopted to analyze seismicity. This analysis was carried out by splitting the study region into cells of size $\mathrm{L} \times \mathrm{L}$, and by calculating the waiting time probability, $\mathrm{P}_{\mathrm{M}, \mathrm{L}}(\mathrm{T})$, if an earthquake occurs in a given cell at $\mathrm{T}_{0}$, while the next earthquake occurs at a time $\mathrm{T}+\mathrm{T}_{0}$.

Following Cristensen et al. (2002), we set $\mathrm{T}_{\text {corr }}$ as the value of an alternative time of the scaling law by:

$\mathrm{T}_{\text {corr }}=\mathrm{TL}^{\mathrm{df}} \mathrm{S}^{-\mathrm{b}}$

where $\mathrm{T}$ is the waiting time; $\mathrm{L}$ is the cell size in degree of the grid; $\mathrm{S}$ is the value of $\mathrm{S} \sim \log _{10} \mathrm{M}$, meaning the size of the rupture area (Utsu 1970); df is the correlation dimension; and $b$ is the $b$-value of the Gutenberg-Richter law.

According to the BCDS model (Christensen et al. 2002), a relationship of a waiting time $\mathrm{T}$, a waiting time probability $\mathrm{P}_{\mathrm{M}, \mathrm{L}}$, and a new variable, $\mathrm{P}_{\text {corr }}$, is created:

$\mathrm{P}_{\text {corr }}\left(\mathrm{T}_{\text {corr }}\right)=\mathrm{T}^{\alpha} \mathrm{P}_{\mathrm{M}, \mathrm{L}}\left(\mathrm{T}_{\text {corr }}\right)$

The following discussions use earthquake data from Taiwan to investigate the relationship between $\mathrm{P}_{\mathrm{M}, \mathrm{L}}(\mathrm{T})$ and $\mathrm{T}$, and $\mathrm{P}_{\text {corr }}$ and $\mathrm{T}_{\text {corr }}$ of Eq. (2) for various situations.

The following parameters for seismicity were obtained before using the BCDS model [Eqs. (1) and (2)]. The exponent of Omori's law, $\alpha$; the critical exponent characteristic of earthquakes of the Gutenberg-Richter law, b; and the distribution of fractal dimensions of epicenters, df. The parameters $\alpha$ and $b$ of each cell were re-calculated and derived by Z-map (Wiemer 2001) and b-value contour lines of Taiwan (Wang 1988) respectively, 1.0 and 0.95 were adopted for the average $\alpha$ and $b$.

\section{DATA}

Earthquakes with a magnitude greater than 2 were selected from the catalogue of the Central Weather Bureau of Taiwan, which had records for earthquakes in the region $21.5^{\circ}-25.5^{\circ} \mathrm{N}$ and $119.5^{\circ}-122.5^{\circ} \mathrm{E}$ from 1973 to 2002 . The analysis was conducted for varying cell sizes, L (degree), and varying cutoff magnitudes, $\mathbf{M}_{c}$, using only earthquakes with magnitude greater than $\mathbf{M}_{\mathrm{c}}$. Earthquake distribution at different depths $(0 \sim 30 \mathrm{~km})$ along this latitude is depicted in Fig. 1.

A correlation dimension, $d_{f}$, proposed by Grassberger and Procaccia (1983) was calculated for all of Taiwan using earthquakes with focal depth less than $30 \mathrm{~km}$ and is shown in Fig. 2. In general, the higher value of $d_{f}$ is located in eastern Taiwan, where most earthquakes occur, and the lower value of $d_{f}$ corresponds to the northwestern parts because it is a region of less earthquake occurrence. An averaged value of $d_{f}=1.37$ is used here.

\section{RESULTS}

Figures. 3a, b, and c show the relationship between $\mathrm{P}_{\mathrm{M}, \mathrm{L}}(\mathrm{T})$ and waiting time, $\mathrm{T}$, for cut-off magnitude, $\mathrm{M}_{\mathrm{c}}=$ 2,3 , and 4, respectively. Different marks in those figures represent different cell sizes. The measure of $\mathrm{P}_{\mathrm{M}, \mathrm{L}}(\mathrm{T})$ is estimated by counting the number of earthquakes at each cell for each waiting time and then dividing by the total number of earthquakes. These charts all show two power-law regimes with different scaling exponents, one from $\mathrm{T}=\sim 40$ to $10^{5}$ seconds and the other with $\mathrm{T}>10^{5}$ seconds. It should be noted that, given a fixed cell size $\mathrm{L}$, the later range of the power- law regime increases as the cut-off magnitude $\mathrm{M}_{\mathrm{c}}$ increases, which is clearly shown in Figs. 3a to c for different $\mathrm{M}_{\mathrm{c}}$. On the other hand, this regime did not vary significantly with cell size.

The behavior of these sets of power-law regime charts reflects the concept of the BCDS model. Next, we analyze the relationship of $P_{\text {corr }}$ and $T_{\text {corr }}$ from Eq. (2) with $\mathrm{M}_{c} \geqq 2$ for varying cell sizes, as illustrated in Fig. 4. The results show that $\mathrm{P}_{\text {corr }}$ is almost constant (the slope is flat) when $\mathrm{T}_{\text {corr }}<1$ (marked by a black vector roughly), but it decreases dramatically with increasing $\mathrm{T}_{\text {corr }}$ when $\mathrm{T}_{\text {corr }}>1$. According to Bak et al. (2002), the conclusion can be drawn that the aftershocks generated after a main shock contribute to the constant part of the scaling relationship, and the fast decay parts are the result of uncorrelated earthquakes. The constant probability density function for a short waiting time can be described by a single power law with exponent zero. It has been rigorously proved by Jonsdottir et al. (2006) with Omori's law. To verify the above conclusion, the data are declustered to remove aftershocks using Z-map (Wiemer 2001) where the declustering procedure is based on Omori's law at a confidence level of 0.95 for observing the next 
event in the sequence with a 10-day maximum look-ahead time (Reasenberg 1985) for clustered events. Figure 5 is the result of removing the aftershocks with the same analysis
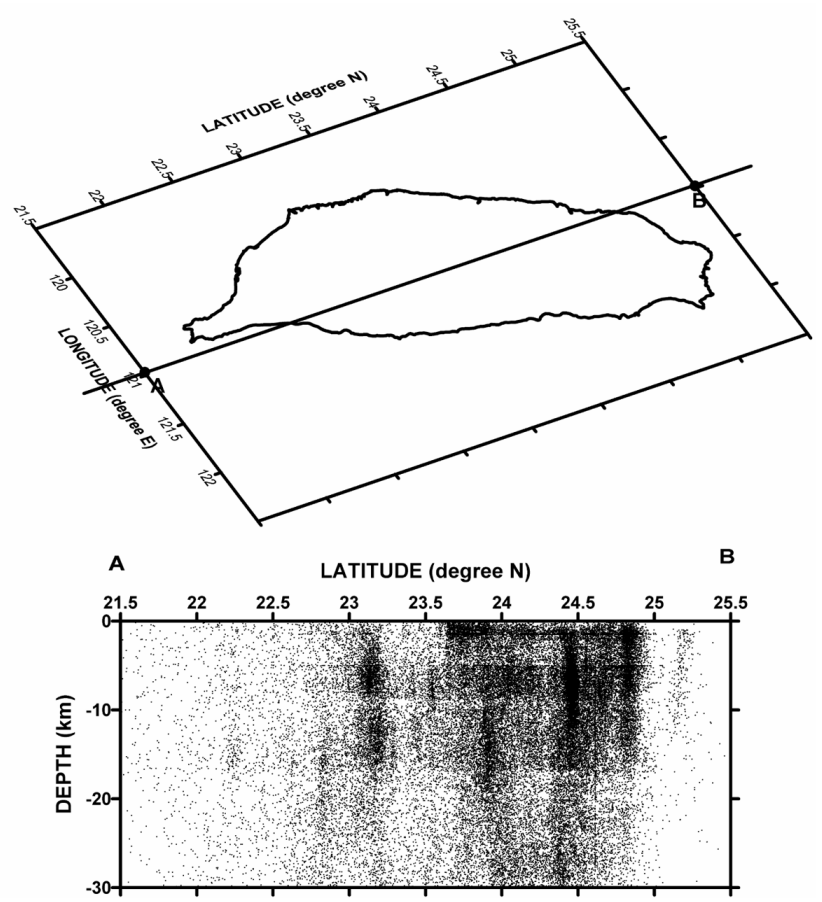

Fig. 1. The distribution of earthquakes at different depths along profile $\mathrm{AB}$. Earthquakes with focal depth less than $30 \mathrm{~km}$ are used to estimate the fractal dimensions.

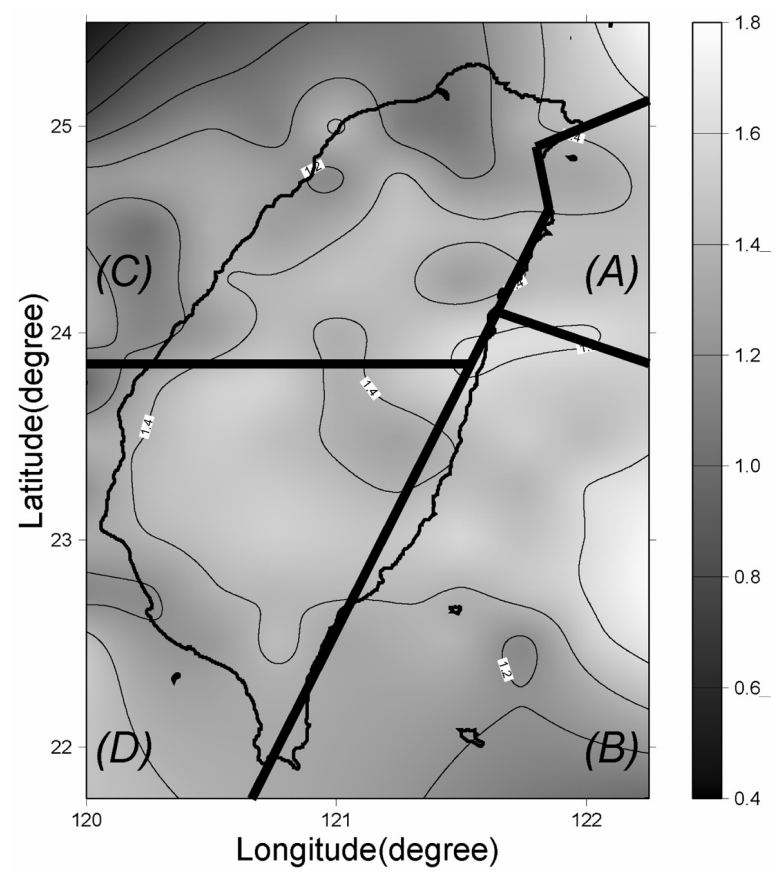

Fig. 2. The distribution of the fractal dimensions of the epicenters of Taiwan. The bold lines separate Taiwan into four areas based on geological boundaries (Wang 1988; Wang and Lee 1996). as in Fig. 4. Comparing Figs. 5 and 4, it is found that the flat part no longer exists (the slope has changed), but the part showing fast decay remains at the same slope rate as in Fig. 5. It is worth noting that $\mathrm{P}_{\text {corr }}$ is directly proportion to $\mathrm{T}_{\text {corr }}$ when $\mathrm{T}_{\text {corr }}<1$ (Fig. 4). This change seems to suggest that the factor representing the effect of the aftershock occurring, $\mathrm{T}^{-\alpha}(\alpha=0.95)$, as shown in Eq. (2) has diminished. This phenomenon was originally observed by Bak et al. (2002); however, it seems to indicate that although the aftershocks are closely related to their main shocks, they are fundamentally different types of phenomena owing to their
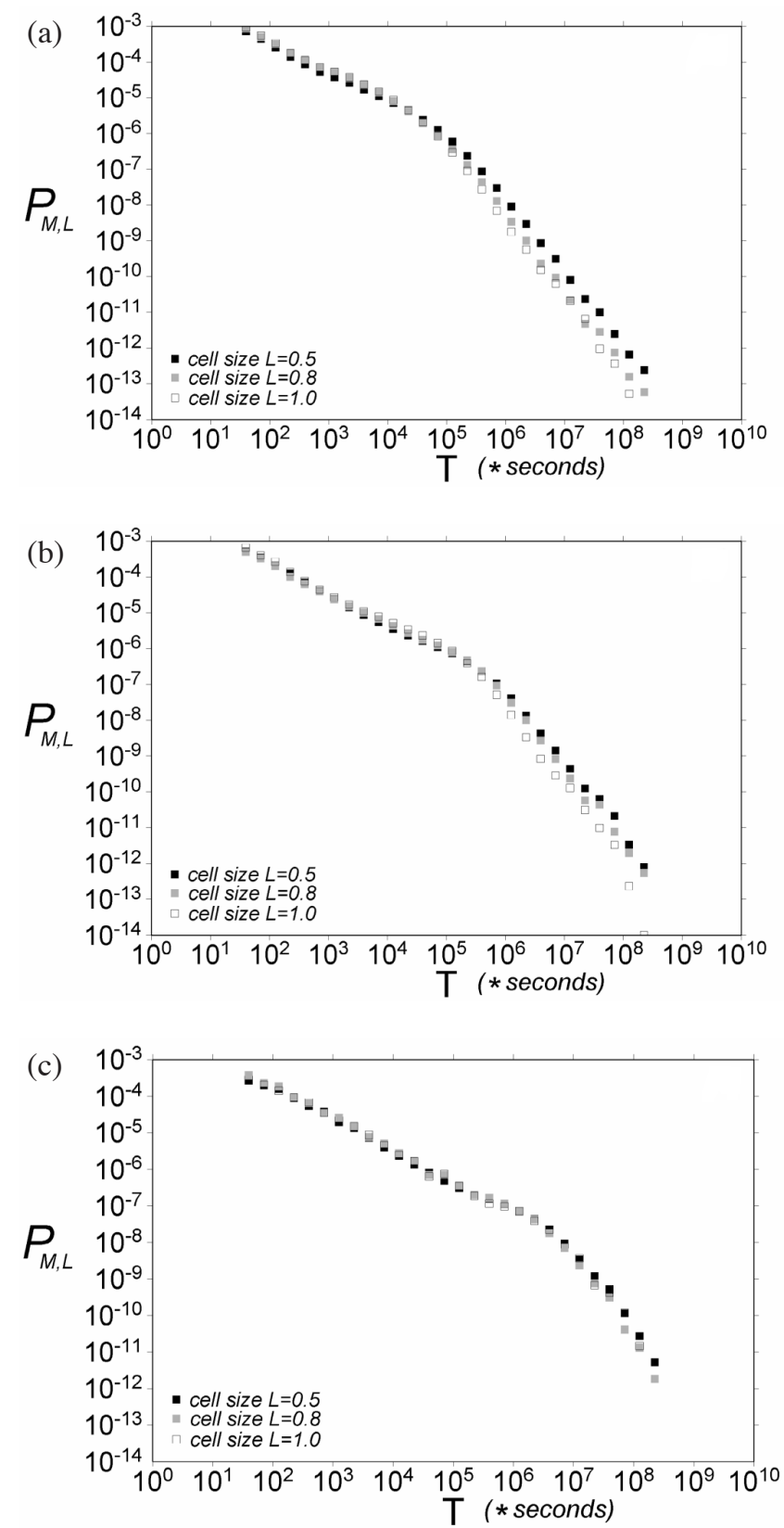

Fig. 3. The log-log plots between $\mathrm{P}_{\mathrm{M}, \mathrm{L}}(\mathrm{T})$ versus $\mathrm{T}$ (waiting time) of different cell sizes for cut-off magnitudes: (a) $\mathrm{M}_{\mathrm{c}}=2.0$, (b) $\mathrm{M}_{\mathrm{c}}=3.0$, and (c) $\mathrm{M}_{\mathrm{c}}=4.0$. 
different mechanisms. To further investigate earthquake distribution, a homogeneous Poisson sequence of earthquakes of cell size $0.5^{\circ}$ at the same time interval $(1973 \sim 2002)$ is generated with the same total number of declustered earthquakes. The analysis shows that a Poisson distribution (star marks) fits declustered earthquake sequences well when $\mathrm{T}_{\text {corr }}>1$. The fitness is not perfect when $\mathrm{T}_{\text {corr }}<1$, which could have been caused by defective declustering; however, they tend to have the same trend. This comparison suggests that the uncorrelated (possibly main shock) sequences are more likely to distribute according to a Poisson distribution.

To verify whether or not the geological complexity of Taiwan influences the scaling relationship of the BCDS model, an analysis is conducted using earthquakes in different geological regions of Taiwan. Four geological regions in Taiwan marked as A, B, C, and D as shown in Fig. 2. This division is not arbitrary; rather it is based on geological boundaries (Wang 1988; Wang and Lee 1996). The number of earthquakes in each region is: 45646 in A; 50164 in B; 21015 in C, and 27327 in D.

Similar analyses with $\mathrm{L}=0.5^{\circ}, \mathrm{M}_{\mathrm{c}}=2$ without removing aftershocks are conducted for these four sub-regions with the corresponding parameters $\left(\alpha, b, d_{f}\right)$ for each region. The results are shown in Fig. 6 (including those for all of Taiwan). By closely examining Fig. 6, it is found that the lines representing the sub-regions A (Northeast), B (Southeast), and D (Southwest) are very close to the line representing the entire area of Taiwan (black solid squares), but for the less earthquake prone region, the sub-region C (Northwest), the curve trends slightly away. The time shift of the kink (marked by a white vector in Fig. 6) for the sub-region $\mathrm{C}$ might be caused by there being less earthquakes (longer waiting time) where the fractal dimension (1.31) is low. And according to Corral and Christensen (2006), it might be caused by a low seismic rate of the sub-region C. Although there are differences between the four sub-regions, they all show a similar pattern in the scaling relationship.

\section{DISCUSSION}

The BCDS model uses a unified scaling law to determine the occurrence of earthquakes from a spatial-temporal perspective. Following the basic principles of this model, we conducted some experiments in order to further prove that this model is truly feasible. As a result, the scaling law reveals two different slopes (Fig. 4), which are widely observed in real and synthetic data (Jonsdottir et al. 2006). When we compare declustering (Fig. 5) with clustering experiments for earthquakes in Taiwan (Fig. 4), it is evident that the constant part $\left(\mathrm{T}_{\text {corr }}<1\right)$, which represents the effects of aftershocks, is significantly different; however, for the fast decaying part $\left(\mathrm{T}_{\text {corr }}>1\right)$, probably representing the effect of main shocks, the same slope remains. Comparison with synthetic data has shown that the declustered data (Fig. 5)

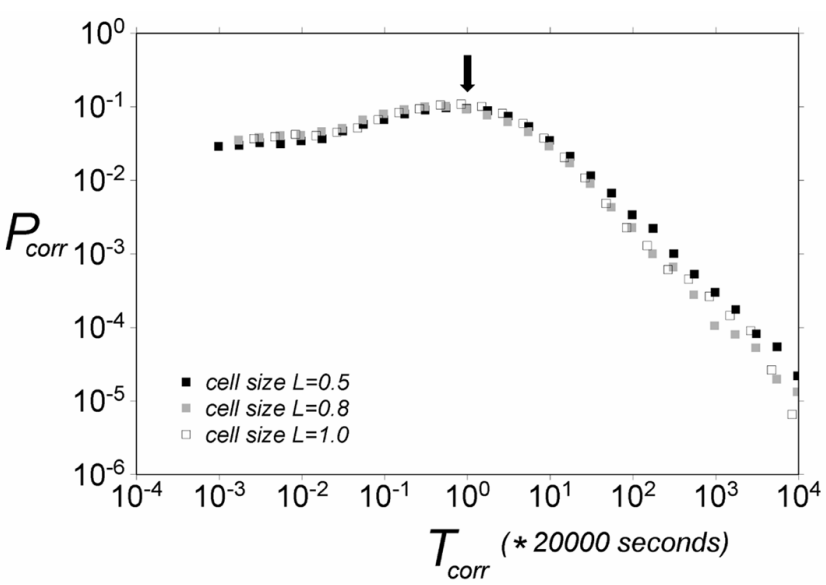

Fig 4. The log-log plots between $\mathrm{P}_{\text {corr }}$ versus $\mathrm{T}_{\text {corr }}$ based on the BCDS model using earthquakes in Taiwan with a cut-off magnitude $\mathrm{M}_{\mathrm{c}}=2.0$ for three values of $\mathrm{L}, 0.5^{\circ}, 0.8^{\circ}$, and $1.0^{\circ}$. The black vector marks the location of the kink at $\mathrm{T}_{\text {corr }} \sim 1$.

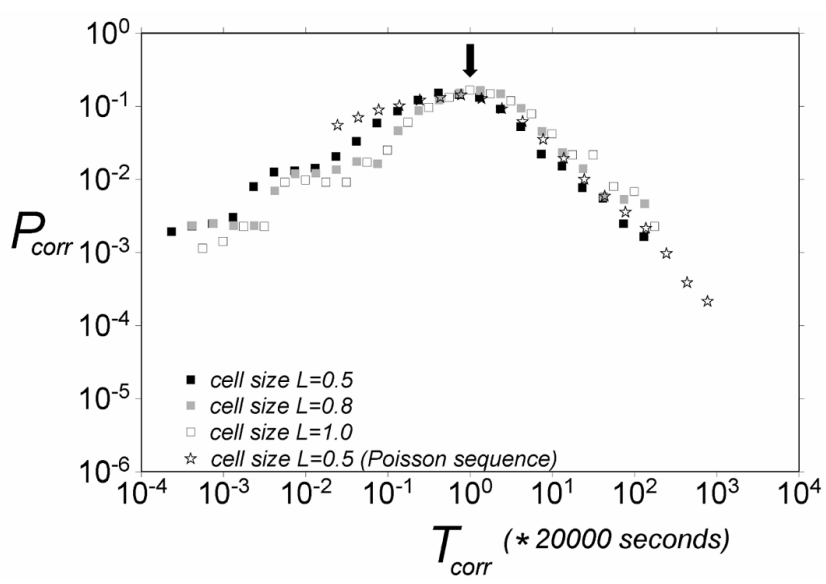

Fig. 5. The log-log plots between $\mathrm{P}_{\text {corr }}$ (removing aftershocks) versus $\mathrm{T}_{\text {corr }}$ based on the BCDS model with a cut-off magnitude $\mathrm{M}_{\mathrm{c}}=2.0$ for three values of $\mathrm{L}$. The star marks, representing the synthetic Poisson sequence, are plotted for comparison. The black vector marks the location of the kink at $\mathrm{T}_{\text {corr }} \sim 1$.

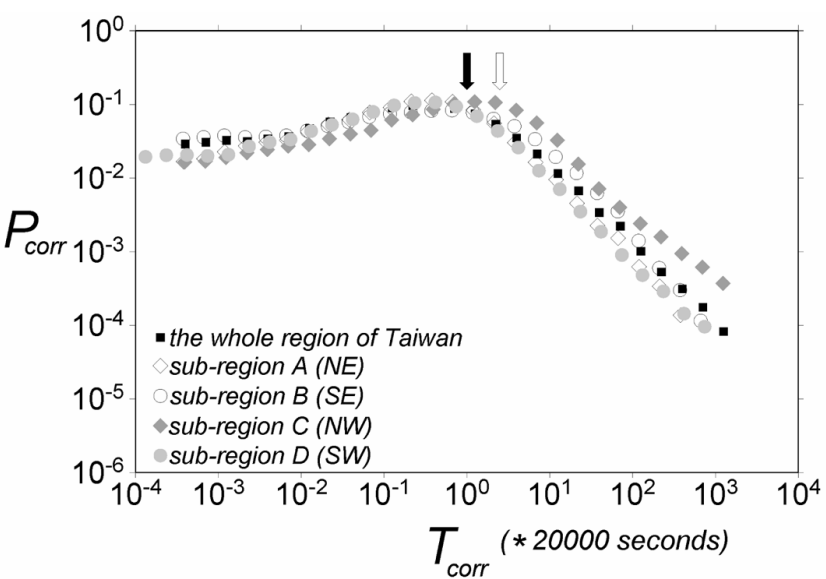

Fig. 6. The log-log plots between $\mathrm{P}_{\text {corr }}$ versus $\mathrm{T}_{\text {corr }}$ based on the BCDS model with cell size $L=0.5^{\circ}$, and cut-off magnitude $M_{c}=2$. The black vector marks the location of the kink for sub-regions $\mathrm{A}, \mathrm{B}$, and $\mathrm{D}$, while the white vector is for sub-region $\mathrm{C}$. 
follows a Poisson distribution, though procedural issues leading to imperfect declustering may result in some deviation in fit. Do uncorrected earthquakes really follow Poisson distributions? Random sequences could any of several distributions; such as: Poisson, Gamma, Weibull, and Beta distributions etc. However, our results seem to indicate a tendency toward the Poisson distribution. In addition, in order to investigate the debate between Lindman et al. (2005) and Corral (2006) about whether or not the distinction of correlated earthquakes (aftershocks) and uncorrelated earthquakes (main shocks) causes a kink at $\mathrm{T}_{\text {corr }}=1$ in the scaling law, we utilized real earthquake data rather than synthetic earthquake data created by simple mathematic modeling, making the exercise both more meaningful and practical.

Expanding the nature of the BCDS model, we prove the existence and validity of a unified scaling law by comparing results between four sub-regions of Taiwan; those being so designated: the Northeast (A), the Northwest (C), Southwest (D), and the Southeast (B). The outcome for subregion, $\mathrm{C}$ (the Northwest), is slightly different to that of the remaining regions. These results show that the BCDS model is more sensitive to the number of earthquakes and spatialtemporal parameters ( $\alpha, b, d_{f}$, seismic rate). In summary, the best way to understand and further interpret self-organized criticality is to choose correct variables and parameters which will allow for complicated occurrences in earthquake spatial-temporal data to collapse into a unified scaling law. Data collapse, a concept widely used in thermal physics by finding a proper parameter [Eqs. (1) and (2)] to change a distribution pattern, clearly verifies the unified scaling law of the BCDS model, and further proves the authenticity of this model that was once assumed to be oversimplified. However, these findings all lead to a bigger challenge; and that is understanding the physics behind the model.

\section{CONCLUSIONS}

Two results were achieved in this paper: (1) Regardless of the size of the cells and the cut-off magnitude, a distinct and unified scaling law is always obtained from the spatialtemporal occurrence of earthquakes in Taiwan. This result once again proves the two key elements of SOC supported by Bak (1988). First, the spatial distribution of events is fractal; second, the size of the avalanche occurs in a power-law relationship. (2) The experiment of comparing declustered and clustered experiments further verifies that earthquakes give two distinct patterns in the BCDS model. A Poisson distribution tends to be a better description for fast decay part result of earthquake sequences. In addition, under real world conditions in a complicated geological region, the existence of a unified scaling law was proven through comparison between four sub-regions of Taiwan. Under appropriate statistical conditions, it was shown that the scaling law representing each region has the same trend.
The findings of this study further increase the reliability and accuracy of the BCDS model. In addition, insights garnered from the use of this model in this paper provide advanced understanding of the spatial-temporal occurrence of earthquakes.

Acknowledgements Two anonymous reviewers provided very constructive comments. This paper greatly benefited from their expert assistance. This research was supported by the National Science Council of Taiwan, ROC under grant NSC 89-2921-M-194-001-EAF.

\section{REFERENCES}

Bak, P., C. Tang, and K. Wiesenfeld, 1988: Self-organized criticality. Phys. Rev. A., 38, 364-374.

Bak, P., K. Christensen, L. Danon, and T. Scanlon, 2002: Unified scaling law for earthquakes. Phys. Rev. Lett., 88, 178501.

Christensen, K., L. Danon, T. Scanlon, and P. Bak, 2002: Unified scaling law for earthquakes. Proc. Natl. Acad. Sci. USA., 99, 2509-2513.

Corral, Á., 2004a: Long-term clustering, scaling, and universality in the temporal occurrence of earthquakes. Phys. Rev. Lett., 92, 108501.

Corral, Á., 2004b: Universal local versus unified global scaling laws in the statistics of seismicity. Physica A., 340, 590-597.

Corral, Á., 2005: Renormalization-group transformations and correlations of seismicity. Phys. Rev. Lett., 95, 028501

Corral, Á. and K. Christensen, 2006: Comment on "Earthquakes Descaled: On Waiting Time Distributions and Scaling Laws”. Phys. Rev. Lett., 96, 109801.

Grassberger, P. and I. Procaccia, 1983: Measuring the strangeness of strange attractors. Physica D., 9, 189208.

Gutenberg, B. and C. F. Richter, 1944: Frequency of earthquakes in California. Bull. Seismol. Soc. Am., 34, 319323.

Jónsdottir, K., M. Lindman, R. Roberts, B. Lund, and R. Bödvarsson, 2006: Modelling fundamental waiting time distributions for earthquake sequences. Tectonophysics, 424, 195-208.

Kagan, Y. Y. and D. D. Jackson, 1994: Long-term probabilistic forecasting of earthquakes. J. Geophys. Res., 99, 13685-13700.

Lindman, M., K. Jonsdottir, R. Roberts, B. Lund, and R. Bödvarsson, 2005: Earthquakes descaled: On waiting time distributions and scaling laws. Phys. Rev. Lett., 94, 108501.

Marsan, D., C. J. Bean, S. Steacy, and J. McCloskey, 2000: Observation of diffusion processes in earthquake populations, and implications for the predictability of seismicity systems. J. Geophys. Res., 105, 28081-28094. 
Okubo, P. G. and K. Aki, 1987: Fractal geometry in the San Andreas fault system. J. Geophys. Res., 92, 345-355.

Omori, F., 1895: On aftershocks of earthquakes. J. Coll. Sci. Imp. Univ. Tokyo, 7, 11-200.

Reasenberg, P., 1985. Second-order moment of Central California seismicity. J. Geophys. Res., 90, 5478-5495.

Utsu, T., 1970: Aftershocks and earthquake statistics (II) - Further investigation of aftershocks and other earthquake sequences based on a new classification of earth- quake sequences. J. Fac. Sci. Hokkaido Univ., Ser. VII, 3, 197-266.

Wang, J. H., 1988: b value of shallow earthquakes in Taiwan. Bull. Seismol. Soc. Am., 78, 1243-1254.

Wang, J. H. and C. W. Lee, 1996: Multifractal measures of earthquakes in west Taiwan. Pure. Appl. Geophys., 146, 131-145.

Wiemer, S., 2001: A software package to analyse seismicity: ZMAP. Seismol. Res. Lett., 72, 373-382. 